\title{
The association between cardiac injury and outcomes in hospitalized patients with COVID-19
}

\author{
Shahrokh Karbalai Saleh ${ }^{1}$. Alireza Oraii ${ }^{2} \cdot$ Abbas Soleimani $^{1} \cdot$ Azar Hadadi $^{3} \cdot$ Zahra Shajari $^{1} \cdot$ Mahnaz Montazeri $^{3}$. \\ Hedieh Moradi $^{4} \cdot$ Mohammad Talebpour $^{5} \cdot$ Azadeh Sadat Naseri $^{1} \cdot$ Pargol Balali $^{2} \cdot$ Mahsa Akhbari $^{6} \cdot$ Haleh Ashraf $^{7,8}$
}

Received: 14 June 2020 / Accepted: 31 July 2020 / Published online: 9 August 2020

(c) Società Italiana di Medicina Interna (SIMI) 2020

\begin{abstract}
In this study, we aimed to assess the association between development of cardiac injury and short-term mortality as well as poor in-hospital outcomes in hospitalized patients with COVID-19. In this prospective, single-center study, we enrolled hospitalized patients with laboratory-confirmed COVID-19 and highly suspicious patients with compatible chest computed tomography features. Cardiac injury was defined as a rise of serum high sensitivity cardiac Troponin-I level above 99th percentile (men: $>26 \mathrm{ng} / \mathrm{mL}$, women: $>11 \mathrm{ng} / \mathrm{mL}$ ). A total of 386 hospitalized patients with COVID-19 were included. Cardiac injury was present among 115 (29.8\%) of the study population. The development of cardiac injury was significantly associated with a higher in-hospital mortality rate compared to those with normal troponin levels $(40.9 \%$ vs $11.1 \%, p$ value $<0.001)$. It was shown that patients with cardiac injury had a significantly lower survival rate after a median follow-up of 18 days from symptom onset $(p \log$-rank $<0.001)$. It was further demonstrated in the multivariable analysis that cardiac injury could possibly increase the risk of short-term mortality in hospitalized patients with COVID-19 $(\mathrm{HR}=1.811, p$-value $=0.023)$. Additionally, preexisting cardiovascular disease, malignancy, blood oxygen saturation $<90 \%$, leukocytosis, and lymphopenia at presentation were independently associated with a greater risk of developing cardiac injury. Development of cardiac injury in hospitalized patients with COVID-19 was significantly associated with higher rates of in-hospital mortality and poor in-hospital outcomes. Additionally, it was shown that development of cardiac injury was associated with a lower shortterm survival rate compared to patients without myocardial damage and could independently increase the risk of short-term mortality by nearly two-fold.
\end{abstract}

Keywords COVID-19 $\cdot$ SARS-CoV-2 $\cdot$ Cardiovascular $\cdot$ Mortality

Electronic supplementary material The online version of this article (https://doi.org/10.1007/s11739-020-02466-1) contains supplementary material, which is available to authorized users.

Shahrokh Karbalai Saleh and Alireza Oraii contributed equally to this work and share co-first authorship

Haleh Ashraf

hashraf@sina.tums.ac.ir

1 Department of Cardiology, Sina Hospital, Tehran University of Medical Sciences, Tehran, Iran

2 Students' Scientific Research Center (SSRC), Tehran University of Medical Sciences, Tehran, Iran

3 Department of Infectious Diseases, Sina Hospital, Tehran University of Medical Sciences, Tehran, Iran

4 Department of Pathology, Sina Hospital, Tehran University of Medical Sciences, Tehran, Iran

\section{Introduction}

In December 2019, first cases of pneumonia of unknown etiology with rapid deterioration into severe respiratory illness emerged in Wuhan, China [1,2]. Genome sequencing studies on respiratory tract samples showed a novel coronavirus

5 Department of Surgery, Sina Hospital, Tehran University of Medical Sciences, Tehran, Iran

6 Sina Hospital, Tehran University of Medical Sciences, Tehran, Iran

7 Research Development Center, Sina Hospital, Tehran University of Medical Sciences, Hasan-Abad Sq., Imam Khomeini St., Tehran 1136746911, Iran

8 Cardiac Primary Prevention Research Center (CPPRC), Cardiovascular Diseases Research Institute, Tehran University of Medical Sciences, Tehran, Iran 
with the phylogenic similarity to severe acute respiratory syndrome coronavirus (SARS-CoV) to be responsible for the outbreak and the virus was then named SARS-CoV-2 [3, 4]. Since then, due to the worldwide spread of the virus, the World Health Organization (WHO) has announced that the new coronavirus disease 2019 (COVID-19) is characterized as a pandemic and global health crisis.

Epidemiologic features and clinical characteristics of patients with COVID-19 are previously assessed in multiple studies with patients primarily presenting with fever, nonproductive cough, and dyspnea [5-7]. However, there are emerging new data regarding possible cardiovascular involvement and myocardial damage with COVID-19. Few case reports have reported patients presenting with severe acute myocarditis $[8,9]$ and pathological findings during autopsy have suggested infiltration of the myocardium by inflammatory mononuclear cells [10]. Previous findings have suggested that the overall prevalence of cardiac injury in patients with COVID-19 is $4.2 \%$ which increases up to $40 \%$ in hospitalized patients [11]. In addition, it is proposed that patients with COVID-19 and concomitant cardiac biomarker elevations during hospitalization might have a worse prognosis [12]. However, data regarding the effect of the SARS-CoV-2 infection on the cardiovascular system remains scarce which increases the need for a comprehensive study to assess clinical outcomes and complications of patients with COVID-19 complicated by newly developed cardiac injury during the hospital course and identify independent predictors of acute cardiac injury in these patients.

In this study, we aimed to investigate the association between the development of acute cardiac injury (defined as elevated serum high sensitivity cardiac Troponin-I (hscTnI) levels above 99th percentile) and short-term mortality as well as poor in-hospital outcomes of hospitalized patients diagnosed with COVID-19 by analysis of the regional data from the Sina Hospital COVID-19 Registry (SHCo-19R).

\section{Methods}

\section{Study design and setting}

The present study was derived from the Sina Hospital COVID-19 Registry (SHCo-19R) which has been described previously in detail regarding study design, diagnostic, and management protocols [13]. In brief, SCHo-19R is an ongoing, prospective, hospital-based registry of patients with COVID-19 presenting to the emergency department of Sina Hospital which is a primary COVID-19 referral center affiliated to Tehran University of Medical Sciences (TUMS) in south Tehran, Iran. The present study is a single-center, retrospective study of a cohort of patients diagnosed with COVID-19 who were hospitalized between March 2020 and
May 2020. This project was approved by the COVID-19 research committee of Sina Hospital and the ethical committee of TUMS (IR.TUMS.VCR.REC.1399.030) and was conducted in accordance with the Declaration of Helsinki [14]. All patients have signed written informed consent forms upon admission prior to enrollment.

\section{Study participants and data collection}

According to WHO interim guidance, consecutive hospitalized patients over 18 years of age with a diagnosis of COVID-19 confirmed by real-time reverse-transcriptase polymerase chain reaction (RT-PCR) of the oropharyngeal swab or endotracheal samples were enrolled in the present study [15]. According to the Iranian national committee of COVID-19, we also enrolled highly suspicious patients including individuals with a compatible chest computed tomography (CT) scan findings including ground-glass opacity alone or ground-glass opacity accompanied by consolidation, not fully explained by volume overload, lobar or lung collapse, or nodules along with the history compatible with COVID-19 [16-19]. Patients who were transferred to other hospitals due to the lack of available beds were excluded from the study.

Patients' demographic data, clinical presentations, laboratory findings, imaging features, hospitalization course data, in-hospital outcomes, and follow-up events were extracted from web-based electronic report forms which were completed by trained staff members during the study period. Management strategies were in accordance with the latest local and international guidelines; hence, these were subject to change during the study period due to rapidly updating literature. Follow-up data regarding vital status, readmission, drug complications, and adverse events were obtained through telephone calls for 1 month after the discharge.

\section{Measures and outcomes}

All patients admitted with suspected COVID-19 underwent electrocardiography (ECG) and diagnostic imaging with a chest CT scan upon admission. In addition, routine laboratory tests were drawn for complete blood count with differential, C-reactive protein (CRP), serum biochemistry, and cardiac biomarkers. Patients with suspected myocarditis underwent further investigation with echocardiography and laboratory tests including lactate dehydrogenase (LDH), creatine phosphokinase (CPK), and pro-brain natriuretic peptide (Pro-BNP). In patients with elevated cardiac biomarkers, at least two serial serum hs-cTnI measurements were obtained and ECG monitoring was performed. Patients with elevated hs-cTnI and evidence of ischemia based on clinical presentation, serial changes of ECG findings, cardiac biomarker measurements or echocardiography were further 
scheduled for the evaluation of concomitant coronary artery disease.

Cardiac injury was defined as an elevated serum level of hs-cTnI above the 99th percentile upper reference limit irrespective of echocardiographic and electrocardiographic features. The 99th percentile of hs-cTnI of our institution's laboratory kit was 26 and $11 \mathrm{ng} / \mathrm{mL}$ for men and women, respectively. Patients were categorized according to the presence or absence of acute cardiac injury and study outcomes included acute respiratory distress syndrome (ARDS), acute kidney injury (AKI), and mortality. ARDS was defined according to the Berlin Definition [20]. AKI was identified based on the serum creatinine level or urine output criteria according to the Kidney Disease: Improving Global Outcomes definitions [21]. However, patients with end-stage renal disease requiring renal replacement therapy were excluded from the mentioned definition for AKI.

\section{Statistical analysis}

Categorical variables are reported as frequency and percentage while continuous variables are expressed as mean \pm standard deviation or median (interquartile range) according to the normality of distribution. Categorical variables were compared using Chi-square or Fisher exact test and continuous variables were compared using independent Student's $t$-test or Mann-Whitney $U$ test, as appropriate. The Kaplan-Meier method was used for plotting survival curves and the significance of differences between patients with and without cardiac injury was assessed using the log-rank test. A multivariable Cox proportional hazards regression analysis was performed to evaluate the effect of acute cardiac injury and other independent risk factors on short-term mortality of hospitalized patients with COVID-19. A univariate logistic regression analysis was performed to identify predictors of acute cardiac injury. Thereafter, variables with a $p$-value of less than 0.05 in the univariate analyses were further assessed in a multivariable logistic regression analysis to identify independent predictors of acute cardiac injury in hospitalized patients with COVID-19. All analyses were performed using SPSS version 22.0 (SPSS Inc, Chicago, Illinois, USA) and a $p$-value of less than 0.05 was considered as the statistical significance level for all analyses.

\section{Results}

A total of 398 patients with a diagnosis of COVID-19 were admitted to the Sina Hospital between March 2020 and May 2020. A total of 12 patients were excluded because they were transferred to other medical centers due to the lack of available beds in our center at the time of admission. At the end, 386 patients with a diagnosis of COVID-19 were included in the analysis and were further categorized according to the presence or absence of acute cardiac injury during hospitalization.

\section{Baseline characteristics and laboratory findings}

The baseline characteristics of patients are shown in Table 1 . Patients had a mean age of $59.46 \pm 15.82$ years and women constituted $38.9 \%$ of the study population. Among these patients, cough was the most frequently seen presenting symptom with a prevalence of $64.5 \%$ followed by dyspnea, fever, malaise/fatigue, and nausea/vomiting with a prevalence of $56.7 \%, 50.8 \%, 30.1 \%$, and $19.2 \%$, respectively. Hypertension was present in 142 patients (36.8\%) as the most common coexisting comorbidity followed by diabetes mellitus (34.5\%) and previous cardiac disease (25.1\%). Among 97 patients with a previous history of cardiovascular disease in our study population, a total of 20 patients had a previous diagnosis of chronic heart failure which constituted $5.2 \%$ of the study population.

A total of 115 patients (29.8\%) had elevated troponin levels during the hospital course indicating the development of acute cardiac injury. Presenting symptoms did not differ significantly between patients with and without cardiac injury. In addition, patients with acute cardiac injury had significantly lower blood oxygen saturation levels $(87.69 \pm 9.25$ vs $90.91 \pm 7.23, p$-value $=0.002$ ) upon presentation. In comparison with patients without cardiac injury, patients with acute cardiac injury during hospitalization were found to be older $(64.98 \pm 15.29$ vs $57.12 \pm 15.48, p$-value $<0.001)$ and have a higher prevalence of underlying comorbidities including hypertension $(51.3 \%$ vs $30.6 \%, p$-value $<0.001)$, previous history of cardiovascular disease (33\% vs $21.8 \%$, $p$-value $=0.021)$, and previous cerebrovascular accident (CVA) or transient ischemic attack (TIA) $(7.8 \%$ vs $2.2 \%$, $p$-value $=0.017)$. Patients with cardiac injury presented with significantly higher levels of inflammatory markers such as white blood cell count, absolute neutrophil count, and C-reactive protein levels at admission (Table 2). In addition, patients with cardiac injury had a higher rate of leukocytosis (33.3\% vs $16.6 \%, p$-value $<0.001)$ and lymphopenia $(44.2 \%$ vs $29.9 \%, p$-value $=0.009$ ) compared to patients without cardiac injury.

\section{Management and in-hospital complications/ outcomes}

Antiviral therapy was the most common treatment strategy in both patients with and without cardiac injury which was administered in 367 patients $(95.1 \%)$ followed by Hydroxychloroquine which was another mainstay of pharmacotherapy in 319 patients (82.6\%) (Table 2). Antiviral therapy predominantly included Lopinavir-Ritonavir (63\%) 
Table 1 Baseline characteristics and radiographic features of hospitalized patients with COVID-19

\begin{tabular}{|c|c|c|c|c|}
\hline & Overall (386) & $\begin{array}{l}\text { Without cardiac } \\
\text { injury (271) }\end{array}$ & With cardiac injury (115) & $p$-value \\
\hline Age, year & $59.46 \pm 15.82$ & $57.12 \pm 15.48$ & $64.98 \pm 15.29$ & $<0.001$ \\
\hline Female & $150(38.9)$ & $104(38.4)$ & $46(40.0)$ & 0.820 \\
\hline \multicolumn{5}{|l|}{ Clinical presentation } \\
\hline Fever & $196(50.8)$ & $143(52.8)$ & $53(46.1)$ & 0.266 \\
\hline Cough & $249(64.5)$ & $176(64.9)$ & $73(63.5)$ & 0.816 \\
\hline Dyspnea & $219(56.7)$ & $149(55.0)$ & $70(60.9)$ & 0.313 \\
\hline Malaise/fatigue & $116(30.1)$ & $77(28.4)$ & $39(33.9)$ & 0.332 \\
\hline Nausea/vomiting & $74(19.2)$ & $53(19.6)$ & $21(18.3)$ & 0.888 \\
\hline Sore throat & $18(4.7)$ & $15(5.5)$ & $3(2.6)$ & 0.294 \\
\hline Systolic blood pressure, $\mathrm{mmHg}$ & $124.54 \pm 22.29$ & $124.84 \pm 22.19$ & $123.88 \pm 22.64$ & 0.727 \\
\hline Heart rate, bpm & $87.67 \pm 16.98$ & $87.21 \pm 16.09$ & $88.71 \pm 18.88$ & 0.478 \\
\hline Blood oxygen saturation, $\%$ & $89.94 \pm 8.02$ & $90.91 \pm 7.23$ & $87.69 \pm 9.25$ & 0.002 \\
\hline \multicolumn{5}{|l|}{ Previous medical history } \\
\hline Diabetes mellitus & $133(34.5)$ & $87(32.1)$ & $46(40.0)$ & 0.160 \\
\hline Hypertension & $142(36.8)$ & $83(30.6)$ & $59(51.3)$ & $<0.001$ \\
\hline Cardiovascular disease & $97(25.1)$ & $59(21.8)$ & $38(33.0)$ & 0.021 \\
\hline CVA/TIA & $15(3.9)$ & $6(2.2)$ & $9(7.8)$ & 0.017 \\
\hline Chronic kidney disease & $16(4.1)$ & $10(3.7)$ & $6(5.2)$ & 0.577 \\
\hline Chronic lung disease & $27(7.0)$ & $15(5.5)$ & $12(10.4)$ & 0.124 \\
\hline Malignancy & $17(4.4)$ & $7(2.6)$ & $10(8.7)$ & 0.013 \\
\hline \multicolumn{5}{|l|}{ Drug history } \\
\hline Statin & $60(15.5)$ & $37(13.7)$ & $23(20)$ & 0.126 \\
\hline Previous ACEI/ARB use & $86(22.3)$ & $52(19.2)$ & $34(29.6)$ & 0.032 \\
\hline Beta blocker & $53(13.7)$ & $34(12.5)$ & $19(16.5)$ & 0.333 \\
\hline Calcium channel blocker & $23(6.0)$ & $13(4.8)$ & $10(8.7)$ & 0.160 \\
\hline Corticosteroid & $14(3.6)$ & $8(3.0)$ & $6(5.2)$ & 0.371 \\
\hline \multicolumn{5}{|l|}{ Radiographic features } \\
\hline Lung involvement & & & & 0.568 \\
\hline Unilateral & $146(37.8)$ & $100(36.9)$ & $46(40.0)$ & \\
\hline Bilateral & $240(62.2)$ & $171(63.1)$ & $69(60.0)$ & \\
\hline
\end{tabular}

Categorical data are expressed as number (percentage) while continuous variables are reported as mean $\pm \mathrm{SD}$

bpm beats per minute, $C V A$ cerebrovascular accident, TIA transient ischemic attack, $A C E I$ angiotensin converting enzyme inhibitor, $A R B$ angiotensin receptor blocker and Oseltamivir (44.6\%). Moreover, patients with cardiac injury more frequently received antibiotic and glucocorticoid therapy compared to patients without cardiac injury. Patients with cardiac injury had a nearly two-fold higher rate of intensive care unit (ICU) admission (35.7\% vs $14 \%$, $p$-value $<0.001)$ and a higher percentage of patients requiring invasive mechanical ventilation for respiratory support (34.8\% vs $9.2 \%, p$-value < 0.001$)$ compared to patients without elevated troponin levels.

Overall, in-hospital mortality was reported in a total of 77 patients $(19.9 \%)$ during the study period. Patients with cardiac injury had a substantially higher in-hospital mortality rate compared to patients without cardiac injury, $40.9 \%$ vs $11.1 \%$, respectively ( $p$-value $<0.001$ ). Presence of acute cardiac injury was also strongly associated with a higher rate of respiratory failure (40\% vs $11.1 \%$, $p$-value $<0.001)$, AKI (30.7\% vs 9.4\%, p-value <0.001), and longer duration of hospital stay $(7.33 \pm 6.19$ vs $5.49 \pm 6.03, p$-value $=0.007)$. In addition, the level of serum hs-cTnI was considerably higher in patients with in-hospital mortality compared to those who survived [median and interquartile range: 40.0 (9.35-164.55) vs 5.3 (1.5-14.38), $p$-value $<0.001]$. Among patients with cardiac injury, a total of $77(67.0 \%)$ patients showed persistently elevated troponin levels during the hospital course while $38(33.0 \%)$ patients had a rising and falling pattern of troponin elevation. Although patients who died during the hospital course had a higher rate of rising pattern of hs-cTnI level compared to those who survived, 
Table 2 Laboratory findings, management, and outcomes of hospitalized patients with COVID-19

\begin{tabular}{|c|c|c|c|c|}
\hline & Overall (386) & Without cardiac injury (271) & With cardiac injury (115) & $p$-value \\
\hline \multicolumn{5}{|l|}{ Laboratory findings, Median (IQR) } \\
\hline White blood cell count, $\times 10^{9} / \mathrm{L}$ & $6.7(5.1-9.45)$ & $6.4(5-8.8)$ & $7.6(5.67-11.32)$ & 0.002 \\
\hline Neutrophils, $\times 10^{9} / \mathrm{L}$ & $4.86(3.48-7.33)$ & $4.49(3.36-6.9)$ & $5.95(4.05-9.05)$ & $<0.001$ \\
\hline Lymphocytes, $\times 10^{9} / \mathrm{L}$ & $1.19(0.91-1.64)$ & $1.23(0.93-1.68)$ & $1.08(0.82-1.58)$ & 0.030 \\
\hline Hemoglobin, g/L & $135(123-150)$ & $137(124-150)$ & $131(118-149)$ & 0.066 \\
\hline Platelets, $\times 10^{9} / \mathrm{L}$ & $191(148-259)$ & $191(148-259)$ & $186(153-257.5)$ & 0.927 \\
\hline C-reactive protein, $\mathrm{mg} / \mathrm{L}$ & $609.5(260.7-1115)$ & $587(219-1040)$ & $695(334.5-1261.5)$ & 0.048 \\
\hline Erythrocyte sedimentation rate, $\mathrm{mm} / \mathrm{h}$ & $44(26-75.25)$ & $42(25-76)$ & $45(26.75-71)$ & 0.447 \\
\hline Urea, $\mu \mathrm{mol} / \mathrm{L}$ & $5410(3820-8150)$ & $4990(3660-7240)$ & $6570(4160-10,360)$ & $<0.001$ \\
\hline Creatinine, $\mu \mathrm{mol} / \mathrm{L}$ & $94.58(78.67-114.92)$ & $91.05(77.79-107.85)$ & $98.12(82.21-131.71)$ & 0.018 \\
\hline $\mathrm{eGFR}, \mathrm{mL} / \mathrm{min} / 1.73 \mathrm{~m}^{2}$ & $69.29(53.83-82.68)$ & $70.91(58.44-82.56)$ & $60.78(44.38-82.86)$ & 0.007 \\
\hline Sodium, mmol/L & $135.5(132.2-138.9)$ & $135.7(132.7-138.8)$ & $134.9(131.4-139)$ & 0.170 \\
\hline Potassium, mmol/L & $4.25(3.95-4.6)$ & $4.2(3.94-4.6)$ & $4.3(3.96-4.6)$ & 0.509 \\
\hline Alanine aminotransferase, U/L & $36(27-52)$ & $34(25-50.25)$ & $37.5(30-53)$ & 0.050 \\
\hline Aspartate aminotransferase, U/L & $51(38-71.25)$ & $48(37.75-69.25)$ & $56.5(39.25-75)$ & 0.028 \\
\hline Lactate dehydrogenase, U/L & $547.5(446-730.5)$ & $531(434-689)$ & $604(469-773)$ & 0.056 \\
\hline \multicolumn{5}{|l|}{ Venous blood gas } \\
\hline $\mathrm{pH}$ & $7.43(7.38-7.47)$ & $7.44(7.40-7.47)$ & $7.43(7.36-7.47)$ & 0.204 \\
\hline $\mathrm{pCO}_{2}, \mathrm{mmHg}$ & $37.45(32.78-42.65)$ & $37.5(33.0-42.55)$ & $36.3(32.05-43.15)$ & 0.518 \\
\hline $\mathrm{HCO}_{3}, \mathrm{mmol} / \mathrm{L}$ & $25.2(21.6-27.4)$ & $25.4(22.25-27.4)$ & $23.8(19.65-27.35)$ & 0.588 \\
\hline \multicolumn{5}{|l|}{ Treatment strategy } \\
\hline Antiviral & $367(95.1)$ & $259(95.6)$ & $108(93.9)$ & 0.607 \\
\hline Lopinavir-ritonavir & $243(63.0)$ & $173(63.8)$ & $70(60.9)$ & 0.645 \\
\hline Oseltamivir & $172(44.6)$ & $127(46.9)$ & $45(39.1)$ & 0.180 \\
\hline Atazanavir & $49(12.7)$ & $33(12.2)$ & $16(13.9)$ & 0.620 \\
\hline Antibiotic & 257 (66.6) & $166(61.3)$ & $91(79.1)$ & 0.001 \\
\hline Glucocorticoid & $68(17.6)$ & $35(12.9)$ & $33(28.7)$ & 0.001 \\
\hline Hydroxychloroquine & $319(82.6)$ & $224(82.7)$ & $95(82.6)$ & 0.991 \\
\hline Interferon & $17(4.4)$ & $13(4.8)$ & $4(3.5)$ & 0.787 \\
\hline ICU admission & $79(20.5)$ & $38(14.0)$ & $41(35.7)$ & $<0.001$ \\
\hline Mechanical ventilation & $65(16.8)$ & $25(9.2)$ & $40(34.8)$ & $<0.001$ \\
\hline \multicolumn{5}{|l|}{ Complications/outcomes } \\
\hline ARDS & $76(19.7)$ & $30(11.1)$ & $46(40.0)$ & $<0.001$ \\
\hline Acute kidney injury & $60(15.9)$ & $25(9.5)$ & $35(31.0)$ & $<0.001$ \\
\hline Length of stay, days $\pm \mathrm{SD}$ & $6.04 \pm 6.13$ & $5.49 \pm 6.03$ & $7.33 \pm 6.19$ & 0.007 \\
\hline In-hospital mortality & $77(19.9)$ & $30(11.1)$ & $47(40.9)$ & $<0.001$ \\
\hline
\end{tabular}

Categorical data are expressed as number (percentage) while continuous variables are reported as mean \pm SD or median (interquartile range) SI conversion factor: to convert lactate dehydrogenase and aminotransferase to microkatal/liter, multiply by 0.0167

$I Q R$ interquartile range; eGFR, estimated glomerular filtration rate, $I C U$ intensive care unit; ARDS, acute respiratory distress syndrome, $S D$ standard deviation

the difference was not statistically significant $(42.6 \%$ vs. $26.5 \%, p$-value $=0.071)$. Among patients who died during hospitalization, seven patients had only cardiac injury and although the hs-cTnI level was higher in patients with cardiac injury accompanied by ARDS and AKI compared to patients with cardiac injury alone, the difference did not reach a significant level $(p$-value $=0.282)$.

\section{Cardiac injury and mortality}

Survival curves were plotted for the two groups of patients with and without cardiac injury after patients were followed for a median duration of 18 days from symptom onset (Fig. 1). The survival curves showed that hospitalized patients with COVID-19 complicated by acute cardiac injury have a considerably higher rate of short-term 
Fig. 1 Short-term mortality of hospitalized patients with COVID-19
Time from symptom onset

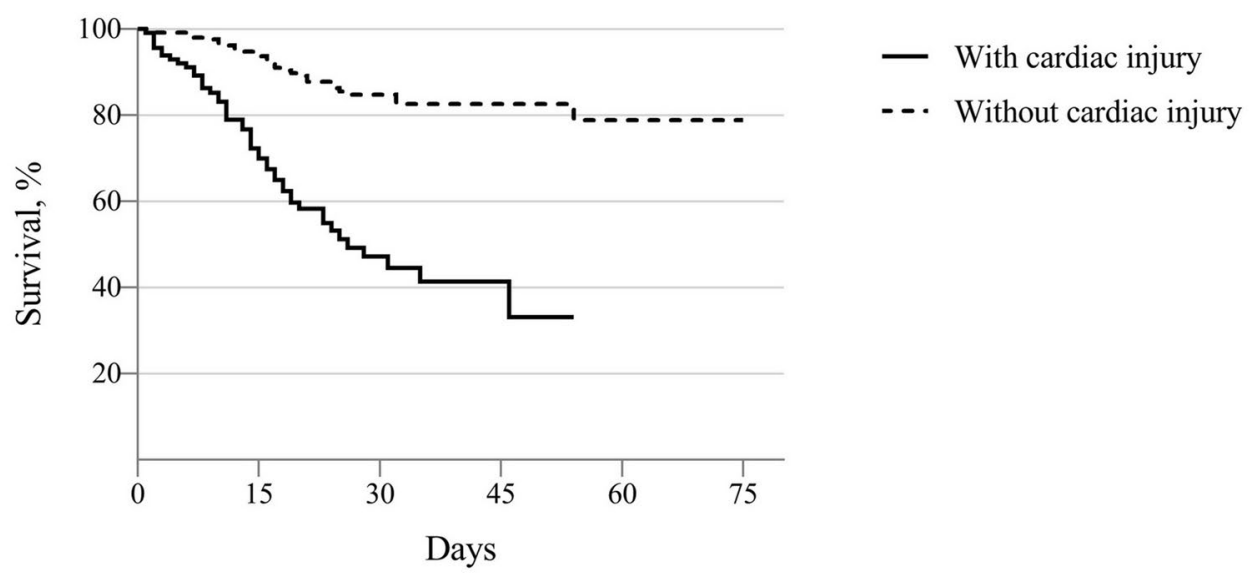

mortality ( $p$ log-rank <0.001). After multivariable Cox proportional hazards regression analysis with adjustment for multiple confounding factors including age, diabetes, cardiovascular disease (including hypertension, coronary heart disease, and congestive heart failure), malignancy, chronic kidney disease, CVA/TIA, previous angiotensin converting enzyme inhibitor (ACEI) or angiotensin receptor blocker (ARB) use, ARDS, and AKI, it was shown that the presence of cardiac injury was significantly associated with an increased risk of short-term mortality and could possibly double the risk of death (HR $=1.811,95 \%$ CI 1.087 to $3.017 ; p$-value $=0.023)($ Table 3$)$. In addition, the model also showed that ARDS (HR $=9.720,95 \%$ CI 4.863 to 19.429; $p$-value $<0.001)$, malignancy $(\mathrm{HR}=5.825,95 \%$ CI 2.568 to $13.213 ; p$-value $<0.001)$, and age $(\mathrm{HR}=1.026,95 \% \mathrm{CI}$ 1.007 to 1.045 ; $p$-value $=0.007)$ are independent risk factors of short-term mortality in hospitalized patients with COVID-19. Univariate Cox proportional hazards regression analyses are shown in Supplementary Table 1.

\section{Predictors of cardiac injury}

A univariate logistic regression analysis was performed to assess the predictors of acute cardiac injury in patients admitted with COVID-19 including baseline characteristics, laboratory findings, and medications (Supplementary Table 2). Afterward, the multivariable model showed that after adjustment for multiple confounding risk factors, blood oxygen saturation $<90 \%(\mathrm{OR}=2.541,95 \% \mathrm{CI}$
Table 3 Multivariable Cox proportional hazards model for short-term mortality in hospitalized patients with COVID-19

\begin{tabular}{lllr}
\hline & Hazard ratio & $\begin{array}{l}\text { 95\% confidence } \\
\text { interval }\end{array}$ & $p$-value \\
\hline $\begin{array}{l}\text { Age (per 1 year } \\
\text { increase) }\end{array}$ & 1.026 & $1.007-1.045$ & 0.007 \\
$\begin{array}{l}\text { Diabetes mellitus } \\
\text { Cardiovascular }\end{array}$ & 1.016 & $0.600-1.721$ & 0.953 \\
$\quad 1.121$ & $0.565-2.226$ & 0.744 \\
$\quad \begin{array}{l}\text { disease } \\
\text { Malignancy }\end{array}$ & 5.825 & $2.568-13.213$ & $<0.001$ \\
$\begin{array}{l}\text { Chronic kidney } \\
\text { disease }\end{array}$ & 1.475 & $0.563-3.863$ & 0.429 \\
CVA/TIA & 1.053 & $0.443-2.500$ & 0.907 \\
$\begin{array}{l}\text { Previous ACEI/ARB } \\
\text { use }\end{array}$ & 0.979 & $0.502-1.909$ & 0.950 \\
ARDS & 9.720 & $4.863-19.429$ & $<0.001$ \\
Acute cardiac injury & 1.811 & $1.087-3.017$ & 0.023 \\
Acute kidney injury & 1.745 & $0.958-3.179$ & 0.069 \\
\hline
\end{tabular}

CVA cerebrovascular accident, TIA transient ischemic attack, ACEI angiotensin converting enzyme inhibitor, $A R B$ angiotensin receptor blocker, $A R D S$ acute respiratory distress syndrome

a Cardiovascular disease includes hypertension, coronary heart disease, or congestive heart failure

1.473 to $4.383 ; p$-value $=0.001)$, leukocytosis $(\mathrm{OR}=2.743$, $95 \%$ CI 1.446 to 5.205 ; $p$-value $=0.002$ ), and lymphopenia $(\mathrm{OR}=2.924,95 \%$ CI 1.632 to $5.238 ; p$-value $<0.001)$ at presentation were independent predictors for development of acute cardiac injury in hospitalized patients with 
COVID-19. In addition, a previous history of cardiovascular disease $(\mathrm{OR}=2.019,95 \%$ CI 1.008-4.045; $p$-value $=0.047)$ and malignancy $(\mathrm{OR}=3.802,95 \%$ CI $1.109-13.035$; $p$-value $=0.034)$ were also significantly associated with an increased risk of developing cardiac injury (Table 4).

\section{Discussion}

The present study showed that patients with COVID-19 complicated by acute cardiac injury were significantly older, had more preexisting comorbidities, and presented with higher levels of inflammatory biomarkers compared to individuals with normal troponin levels. Additionally, we found that patients with COVID-19 and concomitant cardiac injury were at a greater risk of in-hospital poor outcomes including in-hospital mortality, AHRF, and AKI. These patients had a nearly two-fold increased risk of short-term death after a median follow-up of 18 days from symptom onset.

Multiple studies on patients with COVID-19 who required hospitalization have shown that patients at greatest risk of mortality are those who are significantly older and more likely to have underlying comorbidities, especially cardiovascular diseases, hypertension, and diabetes [22, 23]. Moreover, few studies have shown that older patients with previous cardiovascular diseases are more susceptible to severe disease progression sufficient enough for ICU admission [2,24]. These studies have also reported cases with elevated cardiac biomarkers in patients with COVID-19 which were found to be significantly higher in hospitalized patients with severe disease suggesting a possible role of cardiac injury in disease progression. Epidemiological studies have reported that the prevalence of patients with elevated levels of cardiac biomarkers among hospitalized patients with COVID-19 is between 12 and 23\% which can increase up to 46 percent in critically ill patients and non-survivors [2, $7,22,24,25]$. However, there are limited studies on the characteristics of patients with COVID-19 and concomitant elevated cardiac biomarkers and the role of cardiac injury on disease progression and poor outcomes.

In the present study, acute cardiac injury based on elevated hs-cTnI levels above 99th percentile was present in $29.8 \%$ of the study population. Previous large scale studies evaluating patients with COVID-19 have reported that cough, fever, and dyspnea are among the most common presenting symptoms [6]. We found a similar pattern of symptoms at presentation in our study population while there was no significant difference in the prevalence of presenting symptoms between patients with and without cardiac injury. However, despite similar symptoms between the two groups at presentation, patients with cardiac injury presented with more severe acute illness based on a lower blood oxygen saturation level and higher inflammatory biomarkers including white blood cell count and C-reactive protein upon admission. In addition, patients with cardiac injury were found to be older and have more underlying comorbidities compared to patients with normal serum troponin levels which was consistent with the findings of previous studies [26, 27].

In the first reports of hospitalized patients with a diagnosis of COVID-19 in Wuhan, elevated hs-cTnI was present in $7.2-12 \%$ of patients and nearly $80 \%$ of those with myocardial damage required intensive care at $\operatorname{ICU}[2,24]$. Similarly, a recent report of 191 hospitalized patients with COVID-19 showed that a significantly higher rate of cardiac injury (defined as hs-cTnI $>28 \mathrm{ng} / \mathrm{mL}$ ) was present in nonsurvivors [22]. In the present study, the rate of in-hospital mortality as well as other poor in-hospital outcomes including ARDS, AKI, requiring ICU admission, and mechanical ventilation was significantly higher in patients diagnosed with COVID-19 and concomitant cardiac injury compared to those without myocardial damage. Despite having a small sample size and limited number of total deaths, Du et al. showed in a multivariable logistic regression analysis that elevated cardiac troponin I was independently associated with an increased risk of mortality in patients with COVID19 [28]. These findings are supported by a recent study by Shi et al. which showed that $19.7 \%$ of patients admitted with a diagnosis of COVID-19 had cardiac injury during hospitalization and reported that these patients were older, had more underlying comorbidities, and experienced a higher rate of complications during their hospitalization [26]. Consistent with our findings, they also showed that the presence of cardiac injury in COVID-19 could increase the risk of death by nearly four-fold in addition to having a higher rate of in-hospital complications compared to those without cardiac injury. However, the results regarding the incidence of in-hospital death and complications in the mentioned study should be cautiously interpreted as they were limited by the lack of completion of hospital course in multiple patients at the time of publication which might have led to an underestimation of clinical endpoints in both groups of patients with and without cardiac injury. We also found that advanced age, history of malignant disease, and ARDS during hospitalization could be independently associated with the increased risk of short-term mortality. Although borderline insignificant, AKI could also be a possible prognostic factor in hospitalized patients with COVID-19 as these patients are substantially susceptible to dehydration and acute tubular necrosis. These findings point to the fact that severe inflammatory response triggered by COVID-19 could affect multiple organs at the same time with each organ dysfunction contributing differently to the increased risk of mortality.

Previous studies have shown that patients with SARSCoV-2 infection and preexisting cardiovascular disease have an increased risk of severe disease progression which is consistent with our findings suggesting that a history of 
previous cardiovascular disease could possibly double the risk of developing cardiac injury [27, 29]. The study by Guo et al. on 187 patients with COVID-19 showed that $27.8 \%$ of patients had elevated serum levels of cardiac Troponin-T during hospitalization. These patients had a higher rate of mortality compared to those with normal troponin levels $(59.6 \%$ vs $8.9 \%, p$-value $<0.001)$ [27]. The mentioned study also showed that an elevated troponin level in patients with a previous history of cardiovascular disease further increases the rate of mortality up to $69.44 \%$. Interestingly, Guo et al. further reported that patients with elevated serum troponin levels without a previous cardiovascular disease had a higher rate of mortality $(37.50 \%)$ compared to individuals with a history of cardiac disease and a normal troponin level during hospitalization (13.33\%). Based on these findings, early detection and management of patients with cardiac injury/ troponin elevation during hospitalization could have a significant impact on the prognosis of patients with COVID- 19 . In our study, patients with cardiac injury were significantly older with multiple preexisting comorbidities leading to an inherent increased risk for development of cardiac injury by itself. However, we found that patients with blood oxygen saturation $<90 \%$, leukocytosis, and lymphopenia upon presentation are at a greater risk of developing acute cardiac injury in patients admitted with a diagnosis of COVID-19. Higher leukocyte counts and lymphopenia could also be a part of systemic inflammatory response syndrome and might not be directly correlated to cardiac injury. Overall, these findings indicate that patients presenting with severe hypoxia or laboratory markers of inflammation should be closely monitored with cardiac biomarkers for the development of acute cardiac injury during the hospital course.

Although multiple studies have assessed the pathophysiological mechanisms of SARS-CoV-2 infectivity and cell penetration, the exact process of myocardial damage in patients with COVID-19 remains unclear. Previous studies have suggested that SARS-CoV-2 uses viral spike glycoproteins to bind to host cell Angiotensin Converting Enzyme 2 (ACE2) receptors in the lungs [30, 31]. Further reports have suggested that ACE2 receptors are highly expressed on the cell surface of respiratory epithelium as well as other locations such as myocardial cells, gastrointestinal epithelial cells, and proximal tubule cells of kidney [32]. Thus, it is hypothesized that the virus might cause cardiac injury by direct invasion of cardiac cells which is supported by the findings of few case reports of patients with COVID-19 and acute myopericarditis $[8,9]$. However, contradictory findings are reported from autopsy results suggesting scarce interstitial mononuclear infiltrate without substantial myocardial damage in heart tissue [10]. Hence, it is difficult to ascertain if myocardium was primarily targeted by SARS-CoV-2 in patients with COVID-19 and concomitant elevated serum hs-cTnI levels or the developed cardiac injury was secondary to hypoxia and metabolic derangements induced by the ARDS. Although patients with cardiac injury had a higher percentage of patients requiring invasive mechanical ventilation for respiratory support in our study population, lung involvement on chest CT scan and venous gas exchange parameters at presentation were not significantly different between patients with and without elevated troponin levels. Multiple studies on laboratory features of patients with COVID-19 have shown that these patients have increased serum levels of inflammatory biomarkers including C-reactive protein, procalcitonin, inflammatory interleukins, and tumor necrosis factor $\alpha$, especially in patients with severe disease requiring intensive care [2, 22]. Hence, it is suggested that severe acute illness with SARS-CoV-2 could activate a cascade of dysregulated immune response and inflammatory cytokine storm precipitating subsequent hypoxia, increased myocardial oxygen demand, myocardial depression, and multiorgan failure. Overall, the exact mechanism of cardiac injury in patients with COVID-19 is not yet clearly understood and warrants further investigations to clarify the process of myocardial damage in SARS-CoV-2 infection.
Table 4 Multivariable logistic regression analysis of predictors of cardiac injury in hospitalized patients with COVID-19

\begin{tabular}{lllr}
\hline & Odds ratio & $95 \%$ confidence interval & $p$-value \\
\hline Age (per 1 year increase) & 1.018 & $0.998-1.038$ & 0.073 \\
Cardiovascular disease $^{\mathrm{a}}$ & 2.019 & $1.008-4.045$ & 0.047 \\
Malignancy & 3.802 & $1.109-13.035$ & 0.034 \\
CVA/TIA & 2.162 & $0.564-8.289$ & 0.261 \\
Previous ACEI/ARB use & 0.948 & $0.459-1.955$ & 0.884 \\
Blood $\mathrm{O}_{2}$ saturation $<90 \%$ & 2.541 & $1.473-4.383$ & 0.001 \\
WBC $>10,000 \times 10^{9} / \mathrm{L}$ & 2.743 & $1.446-5.205$ & 0.002 \\
Lymphocyte $<1000 \times 10^{9} / \mathrm{L}$ & 2.924 & $1.632-5.238$ & $<0.001$ \\
C-reactive protein & 0.999 & $0.994-1.004$ & 0.706 \\
\hline
\end{tabular}

CVA cerebrovascular accident, TIA transient ischemic attack, ACEI angiotensin converting enzyme inhibitor, $A R B$ angiotensin receptor blocker, $O_{2}$ oxygen, $W B C$ white blood cell

${ }^{a}$ Cardiovascular disease includes hypertension, coronary heart disease, or congestive heart failure 


\section{Limitations}

Our study had some limitations. First, this was a singlecenter study and further large multicenter studies are required to confirm our results. Second, due to the high volume of admissions during the pandemic and limited resources, confirmatory RT-PCR tests were not performed for all patients in our study. However, highly suspicious patients enrolled in the present study were diagnosed according to compatible clinical, laboratory, and radiographic features of COVID-19 based on the latest updates and national guidelines. Third, more specific indices of cardiovascular involvement such as echocardiography, cardiac magnetic resonance imaging, holter monitoring for arrhythmia detection, and cardiac catheterization were not routinely performed for all patients.

\section{Conclusion}

Development of acute cardiac injury in hospitalized patients with COVID-19 was associated with a higher rate of inhospital mortality as well as other poor in-hospital outcomes including ARDS and AKI. In addition, it was shown that cardiac injury could possibly lead to a two-fold increase in the risk of short-term mortality in hospitalized patients with COVID-19. We also found that a previous history of cardiovascular disease, malignancy, severe hypoxia, leukocytosis, and lymphopenia at presentation were significantly associated with a greater risk of developing acute cardiac injury.

Acknowledgements We acknowledge all health-care workers involved in the diagnosis and treatment of patients in Sina Hospital. We are indebted to the Research Development Center of Sina Hospital for its support. The authors are grateful to Mrs. Masoumeh Taleh and Mr. Saeed Hejrani for their help and members of the COVID-19 Crisis Committee of the Sina Hospital for their help and consult.

Authors' contributions SK, AH, MM, HM, MT, and HA contributed to the conception or design of the work. SK, AO, AS, ZS, ASN, PB, $\mathrm{MA}$, and HA contributed to the acquisition, analysis, or interpretation of data for the work. SK, AO, MM, ZS, PB, and MA drafted the manuscript. AS, AH, HM, MT, ASN, and HA critically revised the manuscript. All gave final approval and agree to be accountable for all aspects of work ensuring integrity and accuracy.

Funding This study has been supported by Tehran University of Medical Sciences (Grant number: 99-1-101-47246).

Availability of data and materials The data underlying this article will be shared on reasonable request to the corresponding author.Code availability Not applicable.

\section{Compliance with ethical standards}

Conflict of interest The authors declare that they have no conflict of interest.

Ethics approval This project was approved by the COVID-19 research committee of Sina Hospital and the ethical committee of TUMS (IR. TUMS.VCR.REC.1399.030) and was conducted in accordance with the Declaration of Helsinki.

Informed consent All patients have signed written informed consent forms upon admission prior to enrollment.

Consent for publication Not applicable.

\section{References}

1. Lu H, Stratton CW, Tang YW (2020) Outbreak of pneumonia of unknown etiology in Wuhan, China: the mystery and the miracle. J Med Virol 92(4):401-402. https://doi.org/10.1002/jmv.25678

2. Huang C, Wang Y, Li X, Ren L, Zhao J, Hu Y, Zhang L, Fan G, Xu J, Gu X, Cheng Z, Yu T, Xia J, Wei Y, Wu W, Xie X, Yin W, Li H, Liu M, Xiao Y, Gao H, Guo L, Xie J, Wang G, Jiang R, Gao Z, Jin Q, Wang J, Cao B (2020) Clinical features of patients infected with 2019 novel coronavirus in Wuhan, China. Lancet 395(10223):497-506. https://doi.org/10.1016/s0140 $-6736(20) 30183-5$

3. Zhu N, Zhang D, Wang W, Li X, Yang B, Song J, Zhao X, Huang B, Shi W, Lu R, Niu P, Zhan F, Ma X, Wang D, Xu W, Wu G, Gao GF, Tan W (2020) A novel coronavirus from patients with pneumonia in China, 2019. N Engl J Med 382(8):727-733. https ://doi.org/10.1056/NEJMoa2001017

4. Lu R, Zhao X, Li J, Niu P, Yang B, Wu H, Wang W, Song H, Huang B, Zhu N, Bi Y, Ma X, Zhan F, Wang L, Hu T, Zhou H, Hu Z, Zhou W, Zhao L, Chen J, Meng Y, Wang J, Lin Y, Yuan J, Xie Z, Ma J, Liu WJ, Wang D, Xu W, Holmes EC, Gao GF, Wu G, Chen W, Shi W, Tan W (2020) Genomic characterisation and epidemiology of 2019 novel coronavirus: implications for virus origins and receptor binding. Lancet 395(10224):565-574. https ://doi.org/10.1016/s0140-6736(20)30251-8

5. Chen N, Zhou M, Dong X, Qu J, Gong F, Han Y, Qiu Y, Wang J, Liu Y, Wei Y, Xia J, Yu T, Zhang X, Zhang L (2020) Epidemiological and clinical characteristics of 99 cases of 2019 novel coronavirus pneumonia in Wuhan, China: a descriptive study. Lancet 395(10223):507-513. https://doi.org/10.1016/s0140 $-6736(20) 30211-7$

6. Guan WJ, Ni ZY, Hu Y, Liang WH, Ou CQ, He JX, Liu L, Shan H, Lei CL, Hui DSC, Du B, Li LJ, Zeng G, Yuen KY, Chen RC, Tang CL, Wang T, Chen PY, Xiang J, Li SY, Wang JL, Liang ZJ, Peng YX, Wei L, Liu Y, Hu YH, Peng P, Wang JM, Liu JY, Chen Z, Li G, Zheng ZJ, Qiu SQ, Luo J, Ye CJ, Zhu SY, Zhong NS (2020) Clinical characteristics of coronavirus disease 2019 in China. N Engl J Med. https://doi.org/10.1056/NEJMoa2002032

7. Wang D, Hu B, Hu C, Zhu F, Liu X, Zhang J, Wang B, Xiang H, Cheng Z, Xiong Y, Zhao Y, Li Y, Wang X, Peng Z (2020) Clinical characteristics of 138 hospitalized patients with 2019 novel coronavirus-infected pneumonia in Wuhan, China. JAMA. https ://doi.org/10.1001/jama.2020.1585 
8. Hu H, Ma F, Wei X, Fang Y (2020) Coronavirus fulminant myocarditis saved with glucocorticoid and human immunoglobulin. Eur Heart J. https://doi.org/10.1093/eurheartj/ehaa190

9. Inciardi RM, Lupi L, Zaccone G, Italia L, Raffo M, Tomasoni D, Cani DS, Cerini M, Farina D, Gavazzi E, Maroldi R, Adamo M, Ammirati E, Sinagra G, Lombardi CM, Metra M (2020) Cardiac involvement in a patient with coronavirus disease 2019 (COVID-19). JAMA Cardiol. https://doi.org/10.1001/jamacardio .2020 .1096

10. Xu Z, Shi L, Wang Y, Zhang J, Huang L, Zhang C, Liu S, Zhao P, Liu H, Zhu L, Tai Y, Bai C, Gao T, Song J, Xia P, Dong J, Zhao J, Wang FS (2020) Pathological findings of COVID-19 associated with acute respiratory distress syndrome. Lancet Respir Med 8(4):420-422. https://doi.org/10.1016/s2213-2600(20)30076-X

11. Madjid M, Safavi-Naeini P, Solomon SD, Vardeny O (2020) Potential effects of coronaviruses on the cardiovascular system: a review. JAMA Cardiol. https://doi.org/10.1001/jamacardio .2020 .1286

12. Guo T, Fan Y, Chen M, Wu X, Zhang L, He T, Wang H, Wan J, Wang X, Lu Z (2020) Cardiovascular Implications of fatal outcomes of patients with coronavirus disease 2019 (COVID-19). JAMA Cardiol. https://doi.org/10.1001/jamacardio.2020.1017

13. Talebpour M, Hadadi A, Oraii A, Ashraf H (2020) Rationale and design of a registry in a referral and educational medical center in Tehran, Iran: Sina Hospital Covid-19 Registry (SHCo-19R). Adv J Emerg Med. https://doi.org/10.22114/ajem.v0i0.361

14. World Medical Association Declaration of Helsinki: ethical principles for medical research involving human subjects (2013). JAMA 310 (20):2191-2194. https://doi.org/10.1001/jama.2013.281053

15. World Health Organization (2020) Clinical management of severe acute respiratory infection when COVID-19 is suspected. https:// www.who.int/publications-detail/clinical-management-of-sever e-acute-respiratory-infection-when-novel-coronavirus-(ncov)infection-is-suspected. Accessed 10 May 2020

16. Salehi S, Abedi A, Balakrishnan S, Gholamrezanezhad A (2020) Coronavirus disease 2019 (COVID-19): a systematic review of imaging findings in 919 patients. AJR Am J Roentgenol. https:// doi.org/10.2214/ajr.20.23034

17. Song F, Shi N, Shan F, Zhang Z, Shen J, Lu H, Ling Y, Jiang Y, Shi Y (2020) Emerging 2019 novel coronavirus (2019-nCoV) pneumonia. Radiology 295(1):210-217. https://doi.org/10.1148/ radiol.2020200274

18. Bernheim A, Mei X, Huang M, Yang Y, Fayad ZA, Zhang N, Diao K, Lin B, Zhu X, Li K, Li S, Shan H, Jacobi A, Chung M (2020) Chest CT findings in coronavirus disease-19 (COVID19): relationship to duration of infection. Radiology. https://doi. org/10.1148/radiol.2020200463

19. Health and Treatment Deputy of the Ministry of Health and Medical Education (2020) Guideline for the diagnosis and treatment of COVID-19 in outpatients and inpatients. http://corona.behdasht. gov.ir/files/site1/files/Covid-19_Treatment_Flowcharts_V6.pdf. Accessed 10 May 2020

20. Ranieri VM, Rubenfeld GD, Thompson BT, Ferguson ND, Caldwell E, Fan E, Camporota L, Slutsky AS (2012) Acute respiratory distress syndrome: the Berlin Definition. JAMA 307(23):25262533. https://doi.org/10.1001/jama.2012.5669

21. Kidney disease: improving global outcomes (KDIGO) acute kidney injury work group (2012) KDIGO clinical practice guideline for acute kidney injury. https://kdigo.org/wp-content/uploa ds/2016/10/KDIGO-2012-AKI-Guideline-English.pdf. Accessed 10 May 2020
22. Zhou F, Yu T, Du R, Fan G, Liu Y, Liu Z, Xiang J, Wang Y, Song B, Gu X, Guan L, Wei Y, Li H, Wu X, Xu J, Tu S, Zhang Y, Chen H, Cao B (2020) Clinical course and risk factors for mortality of adult inpatients with COVID-19 in Wuhan, China: a retrospective cohort study. Lancet 395(10229):1054-1062. https ://doi.org/10.1016/s0140-6736(20)30566-3

23. Mehra MR, Desai SS, Kuy S, Henry TD, Patel AN (2020) Cardiovascular disease, drug therapy, and mortality in Covid-19. N Engl J Med. https://doi.org/10.1056/NEJMoa2007621

24. Tian S, Hu N, Lou J, Chen K, Kang X, Xiang Z, Chen H, Wang D, Liu N, Liu D, Chen G, Zhang Y, Li D, Li J, Lian H, Niu S, Zhang L, Zhang J (2020) Characteristics of COVID-19 infection in Beijing. J Infect. https://doi.org/10.1016/j.jinf.2020.02.018

25. Yang X, Yu Y, Xu J, Shu H, Xia J, Liu H, Wu Y, Zhang L, Yu Z, Fang M, Yu T, Wang Y, Pan S, Zou X, Yuan S, Shang Y (2020) Clinical course and outcomes of critically ill patients with SARS-CoV-2 pneumonia in Wuhan, China: a single-centered, retrospective, observational study. Lancet Respir Med. https://doi. org/10.1016/s2213-2600(20)30079-5

26. Shi S, Qin M, Shen B, Cai Y, Liu T, Yang F, Gong W, Liu X, Liang J, Zhao Q, Huang H, Yang B, Huang C (2020) Association of cardiac injury with mortality in hospitalized patients with COVID-19 in Wuhan, China. JAMA Cardiol. https://doi. org/10.1001/jamacardio.2020.0950

27. Zhou P, Yang XL, Wang XG, Hu B, Zhang L, Zhang W, Si HR, Zhu Y, Li B, Huang CL, Chen HD, Chen J, Luo Y, Guo H, Jiang RD, Liu MQ, Chen Y, Shen XR, Wang X, Zheng XS, Zhao K, Chen QJ, Deng F, Liu LL, Yan B, Zhan FX, Wang YY, Xiao GF, Shi ZL (2020) A pneumonia outbreak associated with a new coronavirus of probable bat origin. Nature. https://doi.org/10.1038/ s41586-020-2012-7

28. Du RH, Liang LR, Yang CQ, Wang W, Cao TZ, Li M, Guo GY, Du J, Zheng CL, Zhu Q, Hu M, Li XY, Peng P, Shi HZ (2020) Predictors of mortality for patients with COVID-19 pneumonia caused by SARS-CoV-2: a prospective cohort study. Eur Respir J. https://doi.org/10.1183/13993003.00524-2020

29. Driggin E, Madhavan MV, Bikdeli B, Chuich T, Laracy J, BiondiZoccai G, Brown TS, Der Nigoghossian C, Zidar DA, Haythe J, Brodie D, Beckman JA, Kirtane AJ, Stone GW, Krumholz HM, Parikh SA (2020) Cardiovascular considerations for patients, health care workers, and health systems during the COVID-19 pandemic. J Am Coll Cardiol 75(18):2352-2371. https://doi. org/10.1016/j.jacc.2020.03.031

30. Walls AC, Park YJ, Tortorici MA, Wall A, McGuire AT, Veesler D (2020) Structure, function, and antigenicity of the SARSCoV-2 spike glycoprotein. Cell 181(2):281-292.e286. https:// doi.org/10.1016/j.cell.2020.02.058

31. Chen Y, Guo Y, Pan Y, Zhao ZJ (2020) Structure analysis of the receptor binding of 2019-nCoV. Biochem Biophys Res Commun. https://doi.org/10.1016/j.bbrc.2020.02.071

32. Zou X, Chen K, Zou J, Han P, Hao J, Han Z (2020) Single-cell RNA-seq data analysis on the receptor ACE2 expression reveals the potential risk of different human organs vulnerable to 2019nCoV infection. Front Med. https://doi.org/10.1007/s1168 4-020-0754-0

Publisher's Note Springer Nature remains neutral with regard to jurisdictional claims in published maps and institutional affiliations. 